\title{
Orthonormalization of Substrate and Guided Modes in Effective Resonator Model of Dielectric Multilayer Structure
}

\author{
A. RUDZIŃSKI* \\ Institute of Microelectronics and Optoelectronics \\ Warsaw University of Technology \\ Koszykowa 75, 00-665 Warszawa, Poland
}

(Received May 21, 2007)

\begin{abstract}
In this paper, the orthonormal sets of substrate and guided modes are derived from electromagnetic field distributions of a dielectric multilayer structure, which have been obtained using the effective resonator model, presented in Acta Phys. Pol. A 111, 323 (2007).
\end{abstract}

PACS numbers: 42.70.Qs

\section{Introduction}

The aim of this paper is to present the derivation of orthonormal sets of substrate and guided modes in the effective resonator model of a dielectric multilayer structure, described in [1]. Calculations that can be found here are a continuation of [2], where they have been conducted for radiation modes, therefore, the adopted notation, as well as definitions, are the same as therein.

In the effective resonator model, a plane wave emitted by a source located inside one of the layers of the structure is considered. The layer is treated as a resonant cavity, with reflection coefficients of its right and left boundaries $r_{\mathrm{R}}$ and $r_{\mathrm{L}}$ (which depend on the wave vector $\boldsymbol{k}$ and polarization $\epsilon$ of the plane wave). Distributions of field can then be found by summation of series of reflected plane waves. In particular, in the considered layer, the electromagnetic field is a superposition of two plane waves: one with wave vector $\boldsymbol{k}$, and the second, which could be called reflected, with wave vector $\boldsymbol{k}_{*}$ :

$$
\boldsymbol{k}_{*}=k_{x} \boldsymbol{e}_{x}+k_{y} \boldsymbol{e}_{y}-k_{z} \boldsymbol{e}_{z}
$$

(it is assumed that the $z$ axis is perpendicular to the boundaries of the layer) and

*e-mail: a.rudzinski@elka.pw.edu.pl 
the relation between the amplitude of the reflected plane wave and the emitted one is given by the function

$$
\xi_{\epsilon}(\boldsymbol{k})=\frac{r_{\mathrm{R}}^{*}\left(1-\left|r_{\mathrm{L}}\right|^{2}\right) \mathrm{e}^{-2 \mathrm{i} k_{z} L_{z}}+r_{\mathrm{L}}\left(1-\left|r_{\mathrm{R}}\right|^{2}\right)}{1-\left|r_{\mathrm{L}} r_{\mathrm{R}}\right|^{2}}
$$

with $L_{z}$ being the width of the layer containing the source of radiation.

Orthonormal sets of substrate and guided modes $\boldsymbol{f}_{k \epsilon}$, obeying

$$
\int \mathrm{d}^{3} r n^{2}(\boldsymbol{r}) \boldsymbol{f}_{q \lambda}^{*}(\boldsymbol{r}) \boldsymbol{f}_{k \epsilon}(\boldsymbol{r})=\delta_{\epsilon \lambda} \delta(\boldsymbol{k}-\boldsymbol{q}),
$$

can be constructed in the same way as they have been obtained in the case of radiation modes in [2]. Starting with non-orthonormal modes $\boldsymbol{\psi}_{k \epsilon}$, after calculation of a similar integral, they can be related to the orthonormal modes $\boldsymbol{f}_{k \epsilon}$ by their linear combinations, forming a set of linear equations, which can be easily solved. Normalization of magnetic field distributions $\boldsymbol{f}_{k \epsilon}^{H}$ is then found as

$$
\int \mathrm{d}^{3} r n^{2}(\boldsymbol{r})\left[\boldsymbol{f}_{q \lambda}^{H}(\boldsymbol{r})\right]^{*} \boldsymbol{f}_{k \epsilon}^{H}(\boldsymbol{r})=\frac{\varepsilon_{0}}{\mu_{0}} \int \mathrm{d}^{3} r n^{2}(\boldsymbol{r}) \boldsymbol{f}_{q(-\lambda)}^{*}(\boldsymbol{r}) \boldsymbol{f}_{k(-\epsilon)}(\boldsymbol{r}),
$$

where $(-\mathrm{TE})=\mathrm{TM}$ and $(-\mathrm{TM})=\mathrm{TE}$.

For the non-orthonormal modes

$$
\begin{aligned}
& \int \mathrm{d}^{3} r n^{2}(\boldsymbol{r}) \boldsymbol{\psi}_{q \lambda}^{*}(\boldsymbol{r}) \boldsymbol{\psi}_{k \epsilon}(\boldsymbol{r})=4 \pi^{2} \rho_{\epsilon}(\boldsymbol{k}) \rho_{\epsilon}(\boldsymbol{q}) \\
& \quad \times \delta\left(k_{x}-q_{x}\right) \delta\left(k_{y}-q_{y}\right) \delta_{\epsilon \lambda} \int_{-\infty}^{\infty} \mathrm{d} z n^{2}(z) \boldsymbol{\phi}_{q \epsilon}^{*}(z) \boldsymbol{\phi}_{k \epsilon}(z) .
\end{aligned}
$$

Because of the delta functions, the integral over $z$ on the right-hand side is to be evaluated for $q_{x}=k_{x}$ and $q_{y}=k_{y}$. Using the explicit expressions for $\phi_{k \epsilon}$ the integral becomes

$$
\begin{aligned}
& \int_{-\infty}^{\infty} \mathrm{d} z n^{2}(z) \boldsymbol{\phi}_{q \epsilon}^{*}(z) \boldsymbol{\phi}_{k \epsilon}(z)=\sum_{j=-N_{\mathrm{L}}}^{N_{\mathrm{R}}} n_{(j)}^{2} \\
& \quad \times \int_{(j)} \mathrm{d} \zeta\left[u_{j q \epsilon} \boldsymbol{e}_{q^{(j)} \epsilon} \exp \left(\mathrm{i} q_{z}^{(j)} \zeta\right)+v_{j q_{*} \epsilon} \boldsymbol{e}_{q_{*}^{(j)} \epsilon} \exp \left(-\mathrm{i} q_{z}^{(j)} \zeta\right)\right]^{*} \\
& \quad \times\left[u_{j k \epsilon} \boldsymbol{e}_{k^{(j)} \epsilon} \exp \left(\mathrm{i} k_{z}^{(j)} \zeta\right)+v_{j k_{*} \epsilon} \boldsymbol{e}_{k_{*}^{(j)} \epsilon} \exp \left(-\mathrm{i} k_{z}^{(j)} \zeta\right)\right]
\end{aligned}
$$

where $(j)$ by the integral sign indicates the limits of integration: for $j=-N_{\mathrm{L}}$ from $-\infty$ to 0 , for $j=N_{\mathrm{R}}$ from 0 to $\infty$, and for the remaining layers from 0 to $L_{z}^{(j)}$.

\section{Substrate modes}

A solution of wave equation is a substrate mode, if it is evanescent in exactly one of semi-infinite regions $j=-N_{\mathrm{L}}$ or $j=N_{\mathrm{R}}$. Without loss of generality it can be assumed that $n_{(\mathrm{L})}>n_{(\mathrm{R})}$. A substrate mode is then evanescent in the region $j=N_{\mathrm{R}}$ (for subscripts and superscripts in brackets a shortened notation $\mathrm{R} \equiv N_{\mathrm{R}}$ and $\mathrm{L} \equiv-N_{\mathrm{L}}$ will be used). The $z$ components of the wave vectors $\boldsymbol{k}^{(\mathrm{R})}$ and $\boldsymbol{q}^{(\mathrm{R})}$ in this region are then 


$$
k_{z}^{(\mathrm{R})}=\mathrm{i} \kappa_{z}, \quad q_{z}^{(\mathrm{R})}=\mathrm{i} \eta_{z},
$$

where $\kappa_{z}, \eta_{z}>0$. As shown in Appendix, in this case total reflection occurs, and the reflection coefficient of the right boundary is

$$
r_{\mathrm{R}}=\mathrm{e}^{\mathrm{i} \varphi_{\mathrm{R}}},
$$

where $\varphi_{\mathrm{R}}$ denotes its phase. In the region $j=-N_{\mathrm{L}}$ substrate modes are superpositions of plane waves with $k_{z}^{(\mathrm{L})}, q_{z}^{(\mathrm{L})} \in \mathcal{R}$ and $\left|r_{\mathrm{L}}\right|<1$. In this case, the coupling function (2) is

$$
\xi_{\epsilon}(\boldsymbol{k})=\exp \left(-\mathrm{i} \varphi_{\mathrm{R}}\right) \exp \left(-2 \mathrm{i} k_{z} L_{z}\right),
$$

therefore the coefficient

$$
v_{\mathrm{R} k \epsilon}=0 .
$$

For this reason, the integral over the $j=N_{\mathrm{R}}$ region on the right-hand side of (6) is

$$
\begin{aligned}
& \int_{(\mathrm{R})} \mathrm{d} \zeta u_{\mathrm{R} q \epsilon}^{*} u_{\mathrm{R} k \epsilon} \boldsymbol{e}_{q^{(\mathrm{R})} \epsilon}^{*} \boldsymbol{e}_{k^{(\mathrm{R})} \epsilon} \exp \left(-\left(\kappa_{z}+\eta_{z}\right) \zeta\right) \\
& =\left.u_{\mathrm{R} q \epsilon}^{*} u_{\mathrm{R} k \epsilon} \boldsymbol{e}_{q^{(\mathrm{R})} \epsilon}^{*} \boldsymbol{e}_{k^{(\mathrm{R})} \epsilon} \frac{\exp \left(-\left(\kappa_{z}+\eta_{z}\right) \zeta\right)}{-\left(\kappa_{z}+\eta_{z}\right)}\right|_{0} ^{\infty} .
\end{aligned}
$$

The value of the above expression in the upper limit is 0 , and, as it has been shown in [2], the term resulting from the lower limit cancels out with the term resulting from the upper limit in the region $j=N_{\mathrm{R}}-1$. The integral (6) for substrate modes is very similar to the one obtained for two radiation modes

$$
\begin{aligned}
& \int_{-\infty}^{\infty} \mathrm{d} z n^{2}(z) \phi_{q \epsilon}^{*}(z) \phi_{k \epsilon}(z)=\mathrm{i} n_{(\mathrm{L})}^{2} \lim _{\zeta \rightarrow \infty}\left[\boldsymbol{e}_{q^{(\mathrm{L})} \epsilon}^{*} \boldsymbol{e}_{k}^{(\mathrm{L}) \epsilon}\right. \\
& \quad \times \frac{u_{\mathrm{L} q \epsilon}^{*} u_{\mathrm{L} k \epsilon} \exp \left(-\mathrm{i}\left(k_{z}^{(\mathrm{L})}-q_{z}^{(\mathrm{L})}\right) \zeta\right)^{(\mathrm{L})}-v_{\mathrm{L} q \epsilon} v_{\mathrm{L} k \epsilon}^{*} \exp \left(\mathrm{i}\left(k_{z}^{(\mathrm{L})}-q_{z}^{(\mathrm{L})}\right) \zeta\right)}{k_{z}^{(\mathrm{L})}-q_{z}^{(\mathrm{L})}} \\
& \quad+\boldsymbol{e}_{q_{*}^{*}(\mathrm{~L}) \epsilon} \boldsymbol{e}_{k}^{(\mathrm{L}) \epsilon} \\
& \left.\times \frac{v_{\mathrm{L} q \epsilon} u_{\mathrm{L} k \epsilon} \exp \left(-\mathrm{i}\left(k_{z}^{(\mathrm{L})}+q_{z}^{(\mathrm{L})}\right) \zeta\right)-u_{\mathrm{L} q \epsilon}^{*} v_{\mathrm{L} k \epsilon}^{*} \exp \left(\mathrm{i}\left(k_{z}^{(\mathrm{L})}+q_{z}^{(\mathrm{L})}\right) \zeta\right)}{k_{z}^{(\mathrm{L})}+q_{z}^{(\mathrm{L})}}\right]
\end{aligned}
$$

and, after identifying the delta functions, one obtains

$$
\begin{aligned}
& \int_{-\infty}^{\infty} \mathrm{d} z n^{2}(z) \phi_{q \epsilon}^{*}(z) \phi_{k \epsilon}(z)=n_{(0)}^{2}\left|\frac{k_{z}^{(\mathrm{L})}}{k_{z}}\right| \\
& \quad \times\left[\left(\left|u_{L k \epsilon}\right|^{2}+\left|v_{\mathrm{L} k \epsilon}\right|^{2}\right) \delta\left(k_{z}-q_{z}\right)+2 u_{\mathrm{L} k \epsilon} v_{\mathrm{L} k \epsilon}^{*} \delta\left(k_{z}+q_{z}\right)\right] .
\end{aligned}
$$

The integral (5) for substrate modes can then be written as

$$
\int \mathrm{d}^{3} r n^{2}(\boldsymbol{r}) \boldsymbol{\psi}_{q \lambda}^{*}(\boldsymbol{r}) \boldsymbol{\psi}_{k \epsilon}(\boldsymbol{r})=\rho_{\epsilon}^{2}(\boldsymbol{k}) \delta_{\epsilon \lambda}\left[F_{k \epsilon} \delta(\boldsymbol{k}-\boldsymbol{q})+\tilde{F}_{k \epsilon} \delta\left(\boldsymbol{k}-\boldsymbol{q}_{*}\right)\right],
$$

where 


$$
\begin{aligned}
& F_{k \epsilon}=4 \pi^{3} n_{(0)}^{2}\left|\frac{k_{z}^{(\mathrm{L})}}{k_{z}}\right|\left(\left|u_{\mathrm{L} k \epsilon}\right|^{2}+\left|v_{\mathrm{L} k \epsilon}\right|^{2}\right), \\
& \tilde{F}_{k \epsilon}=8 \pi^{3} n_{(0)}^{2}\left|\frac{k_{z}^{(\mathrm{L})}}{k_{z}}\right| u_{\mathrm{L} k \epsilon} v_{\mathrm{L} k \epsilon}^{*} .
\end{aligned}
$$

\section{Guided modes}

Guided modes are the solutions of wave equation for which both $k_{z}^{(\mathrm{R})}$ and $k_{z}^{(\mathrm{L})}$ are imaginary. In this case, total reflections of the emitted plane wave occur on both boundaries of the layer $j=0$ and the reflection coefficients of the boundaries are

$$
r_{\mathrm{R}}=\mathrm{e}^{\mathrm{i} \varphi_{\mathrm{R}}}, \quad r_{\mathrm{L}}=\mathrm{e}^{\mathrm{i} \varphi_{\mathrm{L}}}
$$

with $\varphi_{\mathrm{R}}$ and $\varphi_{\mathrm{L}}$ being their phases. The mode spectrum (see [3]) is in this case

$$
\rho_{\epsilon}(\boldsymbol{k})=\lim _{|r| \rightarrow 1} \frac{1-|r|^{4}}{1+|r|^{4}-2|r|^{2} \cos \left(\varphi_{\mathrm{tot}}\right)}= \begin{cases}\infty, & \text { if } \cos \left(\varphi_{\mathrm{tot}}\right)=1, \\ 0, & \text { otherwise }\end{cases}
$$

where the total phase $\varphi_{\text {tot }}$ is given by

$$
\varphi_{\mathrm{tot}}=\varphi_{\mathrm{R}}+\varphi_{\mathrm{L}}+2 k_{z} L_{z}
$$

This corresponds to the well-known fact that guided modes do not exist for every possible wave vectors - with the frequency kept constant they form a discrete set. The coupling function (2) in case of a guided mode is expressed as

$$
\begin{aligned}
\xi_{\epsilon}(\boldsymbol{k}) & =\frac{\exp \left(-\mathrm{i} \varphi_{\mathrm{R}}\right) \exp \left(-2 \mathrm{i} k_{z} L_{z}\right)+\exp \left(\mathrm{i} \varphi_{\mathrm{L}}\right)}{2} \\
& =\exp \left(-\mathrm{i} \varphi_{\mathrm{R}}\right) \exp \left(-2 \mathrm{i} k_{z} L_{z}\right)=\exp \left(\mathrm{i} \varphi_{\mathrm{L}}\right),
\end{aligned}
$$

therefore, for the reason explained in Appendix

$$
v_{\mathrm{R} k \epsilon}=v_{\mathrm{L} k \epsilon}=0 \text {. }
$$

The integral over the $j=N_{\mathrm{R}}$ region is again given by (11), and just like in the case of substrate modes, it cancels out with another term. But, for guided modes, the integral over the $j=-N_{\mathrm{L}}$ region has the same form as (11), and it cancels out the same way. This means that for guided modes (6) is equal to 0. However, this conclusion does not apply to the case of equal wave vectors: $\boldsymbol{q}=\boldsymbol{k}$, for which the integral (6):

$$
\begin{aligned}
\int_{-\infty}^{\infty} & \mathrm{d} z n^{2}(z) \phi_{k \epsilon}^{*}(z) \phi_{k \epsilon}(z)=\frac{n_{\mathrm{L}}^{2}\left|u_{\mathrm{Lk \epsilon}}\right|^{2}}{2\left|k_{z}^{(\mathrm{L})}\right|}+\frac{n_{\mathrm{R}}^{2}\left|u_{\mathrm{R} k \epsilon}\right|^{2}}{2\left|k_{z}^{(\mathrm{R})}\right|}+\sum_{-N_{\mathrm{L}}<j<N_{\mathrm{R}}} n_{(j)}^{2} \\
& \times\left\{\left[\left|u_{j k \epsilon}\right|^{2} \exp \left(-\operatorname{Im}\left(k_{z}^{(j)}\right) L_{z}^{(j)}\right)+\left|v_{j k \epsilon}\right|^{2} \exp \left(\operatorname{Im}\left(k_{z}^{(j)}\right) L_{z}^{(j)}\right)\right]\right. \\
& \times \frac{\sinh \left(\operatorname{Im}\left(k_{z}^{(j)}\right) L_{z}^{(j)}\right)}{\operatorname{Im}\left(k_{z}^{(j)}\right)}
\end{aligned}
$$




$$
\left.+2 \operatorname{Re}\left[u_{j k \epsilon} v_{j k \epsilon} e_{k_{*}^{(j)} \epsilon}^{*} \boldsymbol{e}_{k^{(j)} \epsilon} \exp \left(\operatorname{iRe}\left(k_{z}^{(j)}\right) L_{z}^{(j)}\right)\right] \frac{\sin \left(\operatorname{Re}\left(k_{z}^{(j)}\right) L_{z}^{(j)}\right)}{\operatorname{Re}\left(k_{z}^{(j)}\right)}\right\}
$$

can be easily calculated. Normalization of guided modes can be written in the form of (14), where

$$
\begin{aligned}
& F_{k \epsilon}= \begin{cases}4 \pi^{2} \int_{-\infty}^{\infty} \mathrm{d} z n^{2}(z) \phi_{k \epsilon}^{*}(z) \phi_{k \epsilon}(z), & \text { if } \rho_{\epsilon}(\boldsymbol{k}) \neq 0, \\
0, & \text { if } \rho_{\epsilon}(\boldsymbol{k})=0,\end{cases} \\
& \tilde{F}_{k \epsilon}=0,
\end{aligned}
$$

because, for every existing guided mode, the mode spectrum in (14) is infinite anyway. After a proper normalization the guided mode has a finite energy and, though the use of a uniform notation is very convenient, this one can be a bit confusing. In this case, it has to be remembered that the presence of the delta function in the normalization integral is associated only with orthogonality of the modes, and not with their energy, unlike the case of radiation or substrate modes. If only a single guided mode is to be considered, then the delta function should be simply removed.

\section{Summary}

This paper concludes the construction of effective resonator model of a dielectric multilayer structure. The basis of the model has been presented in [1], orthonormalization of radiation modes has been performed in [2], and orthonormalization of substrate and guided modes has been the subject of this paper. The application of an orthonormalization procedure for each type of modes has been shown separately, but it is an obvious observation that two modes of different types are always orthogonal (it follows from the delta functions appearing in (14)). The obtained results allow the use of the effective resonator model in construction of models of various physical phenomena concerning interaction of light and matter (e.g. spontaneous or stimulated emission) in a dielectric multilayer structure.

\section{Appendix}

\section{Total reflection from a dielectric multilayer}

Let us consider a plane wave in a dielectric medium with refractive index $n_{(0)}$, next to a dielectric multilayer structure, built of $N-1$ layers, and a dielectric medium with index of refraction $n_{(N)}<n_{(0)}$ behind it. Without loss of generality, the amplitude of the wave can be chosen to be 1 . In this case there is also present a reflected plane wave with amplitude $r$, which is the reflection coefficient. It is a well-known fact that for the angle of incidence greater than the total reflection angle 


$$
\theta_{\mathrm{T}}=\arcsin \frac{n_{(N)}}{n_{(0)}}
$$

the reflection coefficient has

$$
|r|=1 \text {. }
$$

It can be very easily shown in the case of only two dielectric media. Using the notation adopted in the effective resonator model [1,2], the relation between the incident, reflected and transmitted (with amplitude $t$ ) wave is

$$
m_{1,0}\left(\begin{array}{l}
1 \\
r
\end{array}\right)=\left(\begin{array}{c}
t \\
0
\end{array}\right)
$$

If the continuity condition matrix is written explicitly, then a very simple calculation leads to (A.2).

Let $N$ be odd and every layer with even index $j$ (i.e. $j=0,2, \ldots, N-1$ ) have the refractive index $n_{(0)}$, for odd $j$ the refractive index being $n_{(j)}$. For even $j$, the relation between the amplitudes of waves in the $j$-th and $(j+2)$-th layer is given by the translation matrix $M_{j}$, because these layers have the same index of refraction. Therefore, the matrix relating the amplitudes of waves in $(N-1)$-th layer and the dielectric with $j=0$ is the product

$$
m_{N-1,0}=M_{\frac{N-1}{2}} \ldots M_{1} M_{0} .
$$

Diagonal and antidiagonal of a translation matrix contain complex numbers which are their own conjugates and determinant of this matrix is unity

$$
M_{j}=\left(\begin{array}{cc}
D_{j}^{*} & B_{j} \\
B_{j}^{*} & D_{j}
\end{array}\right), \quad \operatorname{det}\left(M_{j}\right)=\left|D_{j}\right|^{2}-\left|B_{j}\right|^{2}=1
$$

A product of two matrices with these properties also has the same properties. Thus, matrix $m_{N-1,0}$ can be written as

$$
m_{N-1,0}=\left(\begin{array}{ll}
D^{*} & B \\
B^{*} & D
\end{array}\right) .
$$

If the $(N-1)$-th layer ends with a mirror with reflection coefficient $r^{\prime}$, then the relation between amplitudes of plane waves in this layer and the incident and reflected plane wave in the $j=0$ layer is

$$
\left(\begin{array}{c}
a_{N-1} \\
r^{\prime} a_{N-1}
\end{array}\right)=\left(\begin{array}{cc}
D^{*} & B \\
B^{*} & D
\end{array}\right)\left(\begin{array}{c}
1 \\
r
\end{array}\right)
$$

Solving for $r$, one obtains

$$
r=\frac{D^{*} r^{\prime}-B^{*}}{D-B r^{\prime}}
$$

For angles greater than $\theta_{\mathrm{T}}\left|r^{\prime}\right|=1$ and $|r|=1$ as well. Indeed, if $r^{\prime}=\left|r^{\prime}\right| \mathrm{e}^{\mathrm{i} \varphi^{\prime}}$ :

$$
r=\frac{D^{*}\left|r^{\prime}\right| \exp \left(\frac{\mathrm{i} \varphi^{\prime}}{2}\right)-B^{*} \exp \left(-\frac{\mathrm{i} \varphi^{\prime}}{2}\right)}{D \exp \left(-\frac{\mathrm{i} \varphi^{\prime}}{2}\right)-B\left|r^{\prime}\right| \exp \left(\frac{\mathrm{i} \varphi^{\prime}}{2}\right)} .
$$

If $\left|r^{\prime}\right|=1$ then the numerator is the complex conjugate of the nominator and therefore $|r|=1$. 
With this knowledge, a simple trick can be used to prove (A.2) for a random composition of layers between the two media. So far, the discussed structure has not been general. However, the widths of layers with fixed refractive indices (i.e. with $j=2,4, \ldots, N-1$ ) can be set to 0 , then the structure built of the remaining layers has no more constraints, yet the obtained conclusion still holds (if a "virtual" layer with width 0 is placed between any two layers, it does not change the relation between the amplitudes in those layers). Therefore, $|r|=1$ for any dielectric multilayer structure if the angle of incidence is greater than $\theta_{\mathrm{T}}$.

\section{References}

[1] A. Rudziński, Acta Phys. Pol. A 111, 323 (2007).

[2] A. Rudziński, Acta Phys. Pol. A 112, 495 (2007).

[3] A. Rudziński, A. Tyszka-Zawadzka, P. Szczepański, Proc. SPIE 5950, 59501A (2005). 